\title{
Application of Remote Ultrasound in COVID-19 Isolated Intensive Care Unit
}

\author{
Xingxi Lin, MD ${ }^{a, 1}$, Jianqiu Hu, MD ${ }^{a, 1}$, Liuqiong Ren, MD ${ }^{a}$, Yuqing Huang, MD ${ }^{a}$, Dudu Wu, MD ${ }^{a}$, Shiyue Zhao, \\ MD ${ }^{a}$, Yuanyuan Zhao, MD ${ }^{a}$, Jie Liu, MD ${ }^{b}$, Xuan Zhou, MD ${ }^{b}$, Weihua Li, MD ${ }^{c}$, Ming Zhang, MD ${ }^{c}$, Bingqi \\ Zhang, MD ${ }^{a}$, Haidan Lin, MD ${ }^{a}$, Shengzheng $W u, M D^{a, *}$, Faqin Lv, MD ${ }^{a, d, *}$ \\ ${ }^{a}$ Department of Ultrasound, Hainan Hospital of PLA General Hospital, Sanya, China; ${ }^{b}$ Department of Emergency, Hainan Hospital of \\ PLA General Hospital, Sanya, China; ${ }^{c}$ Sansha People's Hospital, Sansha, China; ${ }^{d}$ Department of Ultrasound, The First Medical Center \\ of PLA General Hospital, Beijing, China \\ Received April 01, 2020; revision received April 15, 2020; accepted April 16, 2020
}

Objective: To investigate the application of remote ultrasound in COVID-19 isolation ward.

\begin{abstract}
Methods: Two patients with suspected COVID-19 were admitted to the isolation ward in critical condition. In the isolation ward, the intensive care unit (ICU) physician used ultrasound to evaluate patients' heart, lung, abdomen, blood volume, and lower limb veins, guided by an ultrasound expert in remote.

Results: (1) Under the guidance of the ultrasound expert, the ICU physician successfully performed ultrasound examination and gathered clear images. (2) The main manifestations of the patients' lung lesions were multiple B3-lines in both lungs fused to "white lung" and pulmonary consolidation in the subpleural area. A small amount of effusion was observed in the bilateral costal diaphragmatic angle and left lower extremity vein thrombosis was found in patient No.1. Ultrasound was also used to assess the inner diameter and respiratory variability of the inferior vena cava for adjusting fluid supplementation. Patient No.1's condition was severe with rapidly progressing pulmonary lesions. After treatment, ultrasound showed improvement in pneumonia and disappearance of pleural effusion and the left lower extremity vein thrombosis. (3) There was a high degree of consistency between ultrasound and chest computerized tomography (CT) findings in two patients.
\end{abstract}

Conclusion: The application of remote ultrasound can reduce the risk of cross-infection and save the personal protective equipment in the COVID-19 outbreak. Ultrasound experts can also provide real-time guidance for ICU physician to maintain a good quality of patient care and receive high-quality images for accurate diagnosis.

Key words: Remote ultrasound; Intensive care unit; COVID-19; Infectious

Advanced Ultrasound in Diagnosis and Therapy 2020;02:123-127

DOI: $10.37015 / A U D T .2020 .200037$

$\mathrm{T}$ The clinical manifestations of COVID-19 mainly include fever, dry cough and fatigue, and the condition could deteriorate rapidly. Most of the critically ill patients started with dyspnea and/ or hypoxemia 1 week after the symptoms' onset, then rapidly progress to acute respiratory distress syndrome, septic shock, refractory metabolic acidosis, coagulation dysfunction, and multi-organ failure. According to "COVID-19 therapy (Seventh trial version)" issued by Chinese health committee, the diagnosis of COVID-19 relies mainly on reverse transcription polymerase chain reaction (RT-PCR) and serological detection. Positive

\footnotetext{
${ }^{1}$ Co-first authors

* Corresponding authors: Department of Ultrasound, Hainan Hospital of PLA General Hospital, Sanya 572013, China

e-mail:lvjin8912@163.com (FQLv) or emailwsz@126.com (SZWu)

2576-2508/O AUDT 2020 • http://www.AUDT.org

This is an open-access article distributed under the terms of the Creative Commons Attribution 4.0 International license, which permits unrestricted use, distribution and reproduction in any medium provided that the original work is properly attributed.
} 
serological test should meet $1 / 3$ criteria: 1) positive coronavirus specific antibody of $\operatorname{IgM}$ and $\mathrm{IgG} ; 2$ ) serum coronavirus specific IgG antibody changed from negative to positive; 3 ) recovery phase antibody titer is $\geqslant 4$ times of the acute phase titer. Of note, virus detection is prone to be false negative, and the processing time is long [1].

Imaging examination can be used as an important auxiliary means for the diagnosis of COVID-19. It is fast and convenient to clarify the scope and degree of pulmonary lesions to guide clinical treatment. At present, computerized tomography (CT) is the main imaging modality for the clinical diagnosis of COVID-19. However, in the outbreak of COVID-19, the large number of patients makes CT equipment relatively in short supply. Moreover, CT exam is not suitable for patients with severe COVID-19 who are difficult to be transferred out of the unit, cannot cooperate with breathing, or need multiple re-examinations [2], and its disinfection is complex and time-consuming. In this condition, bedside ultrasound, which is quick to perform, portable, repeatable, non-ionizing, has been playing a crucial role in performing real-time pulmonary examination of severe COVID-19 patients. In addition, ultrasound can simultaneously evaluate heart, liver, kidney, pancreas, and lower limb veins [3], and it could also inform the clinicians whether there is insufficient, excessive fluid, or any changes of the blood/fluid volume.

Because of the shortage of personal protective equipment (PPE) and high infectious rate among patients and healthcare workers during the pandemic, radiologists were facing the challenge of satisfying the clinical need. At the same time, many ICU clinicians lack the related ultrasonic knowledge and operation skills, so the use of bedside ultrasound in the isolation ward is largely limited and delayed. This article introduced our own experience of applying remote ultrasound for two patients with critically pneumonia and the outcomes.

\section{Materials and Methods}

\section{Subjects}

Two critically ill patients with severe pneumonia. Case 1: A 53-years-old female was admitted to our hospital due to the expectoration of yellowish-white sticky sputum and a fever of $37.6{ }^{\circ} \mathrm{C}$. Her sputum was tested negative for coronavirus 3 days after she left Hubei Province, China. However, IgG antibody was positive. Case 2: A 59-years-old male had repeated high fever in January without cough or sputum. In an outside hospital, his pulse oxygen had significant dropped with shortness of breath, dyspnea, and other symptoms. After conventional treatment, his dyspnea was not significantly relieved, and his other symptoms got aggravated. His pulse oxygen was at $70 \%$ in room air. The coronavirus nucleic acid test before admission was negative. After that, the patient was transferred to the isolated ICU of our hospital with the diagnosis of "severe pneumonia".

\section{CT examination}

The two patients' chest CT examinations showed diffuse ground glass shadows distributed in both lungs, which were similar to the typical pulmonary manifestations of COVID-19.

\section{Remote ultrasound}

(1) Ultrasonic machine: Clover 70 bedside ultrasonic instrument (manufactured by Wisonic, Shenzhen China). Disinfection was done before and after to avoid cross infection.

(2) Probe selection and operation: Convex array probe frequency is $2 \sim 5 \mathrm{MHz}$, phased array probe frequency is $2.5 \sim 8 \mathrm{MHz}$, and linear array probe frequency is 7.5 10MHz. This machine adopts the synchronous mode of remote ultrasonic data transmission. While the patient end is operating, the data is collected and transmitted to the ultrasound experts at remote end synchronously [4]. The clinician equipped with PPE will collect images from echocardiography, inferior vena cava, and pulmonary ultrasound of patients, upload them through the network, and then consult the ultrasound experts. The ultrasound experts can guide the clinician at the patient end guide and receive realtime ultrasound images. The clinician at the patient end can consult the ultrasound experts about the patient position, probe position, and machine adjustment, etc. to get clear and accurate ultrasound images. All the data collected were recorded in the ultrasonic record sheet of critically ill patients.

\section{(3) Critical ultrasound assessment}

A clinician with inadequate ultrasound working experience stayed at the patient end, and his ultrasound examination was guided by an ultrasound expert remotely (Fig. 1). Before entering the isolation ward, the patient-side clinician and the remote ultrasound expert tested the remote system in advance, and the focus of the target site was evaluated by standardized position fixation.

Exam details: unilateral six-zone ultrasound examination was conducted for the lung [3], followed by the examination of inner diameter and respiratory variation of the inferior vena cava, liver, pancreas, bilateral kidneys, and abdominal cavity. The phasedarray probe was then used for the examination of the heart, and the linear array probe was lastly used for the examination of the lower extremity vein. 

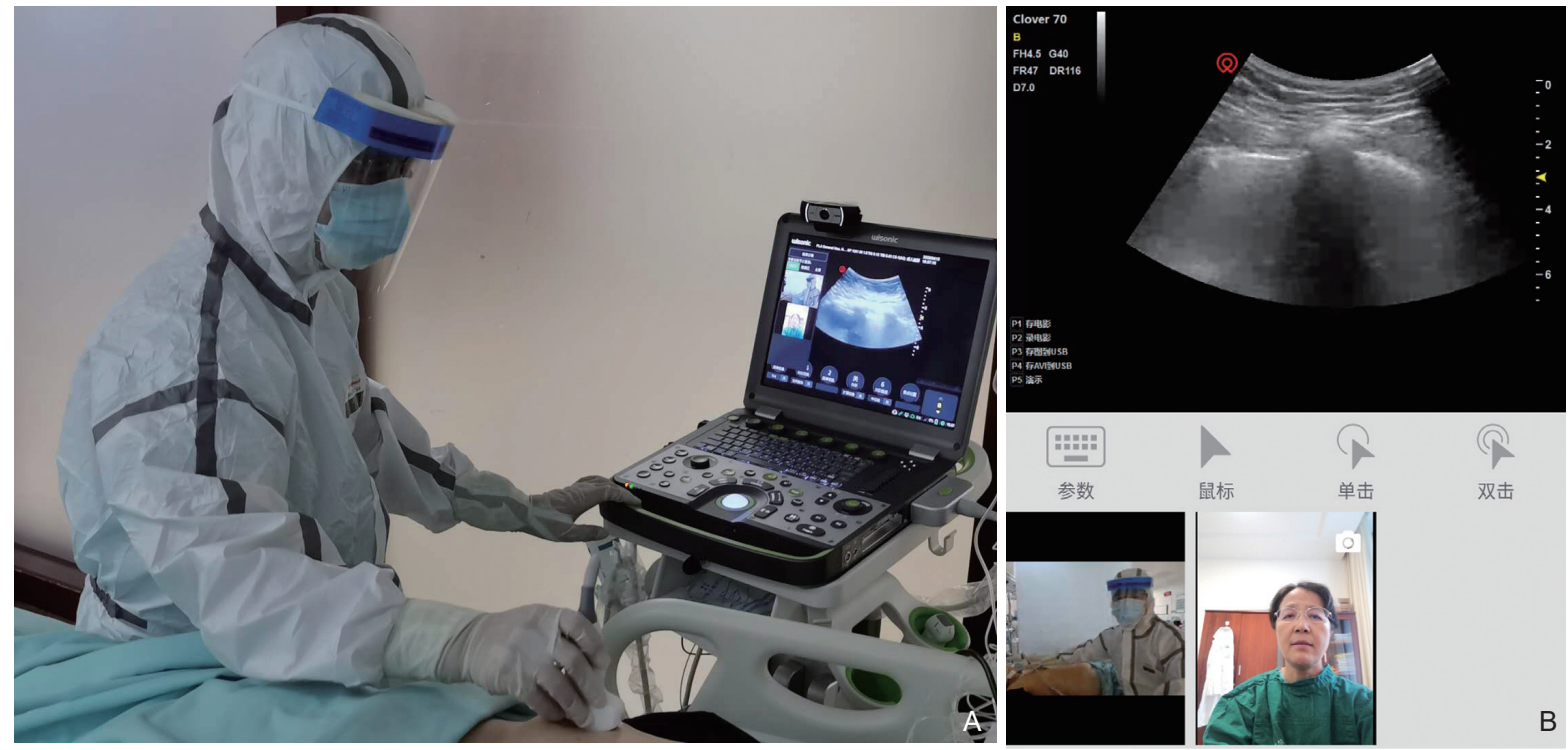

Figure 1 Remote end and consultation end of remote ultrasonography. (A) In the isolation ward, a junior intensive-care physician was using ultrasound to examine unconscious patients at bedside; (B) Ultrasound experts stayed at the consultation end, providing remote ultrasound guidance to doctors in the isolation ward.

\section{Laboratory examination:}

Laboratory test included as follows: (1) RTPCR、IgM and IgG antibody; (2) blood routine; (3) inflammatory indicators (leukocyte, c-reactive protein and procalcitonin); (4) D-Dimer.

\section{Results}

After the treatment of mechanical ventilation, prophylaxis antibiotics, steroids, continuous renal replacement therapy, endoscopic tracheal sputum aspiration and lavage, both the remote ultrasound and CT examination observed gradual improvement in patients' lung condition and the general physical condition. Later, two patients were ruled out COVID-19.

\section{Remote ultrasonic examination}

In case one, ultrasound initially showed the diffusely distributed fused B3-lines in both lungs, absent A-lines and lung sliding sign. Then, B3-lines started to decrease and B7-line occurred. The subpleural consolidation region was no longer widely distributed, and it gradually limited to the 4th-6th zones in both lungs, and finally disappeared. In the end, A-lines re-appeared in the 12 zones of both lungs, with normal pleural activity and no pleural effusion (Fig. 2). There were no abnormalities in cardiac morphological structure, function and hemodynamics. On the 3rd day, left lower extremity vein thrombosis was observed. During this period, liver enzymes and trypsin were slightly increased, but no related abnormalities found in ultrasound examination.

In case two, ultrasound findings were similar to case one. Imaging initially showed diffused B3-line fusion, disappeared A-line and lung sliding signs. Then B3-line decreased, and B7-line occurred. Later, A-line and lung sliding sign re-appeared. No abnormality was found in the heart, pancreas, spleen, gallbladder, kidneys and abdominal cavity.
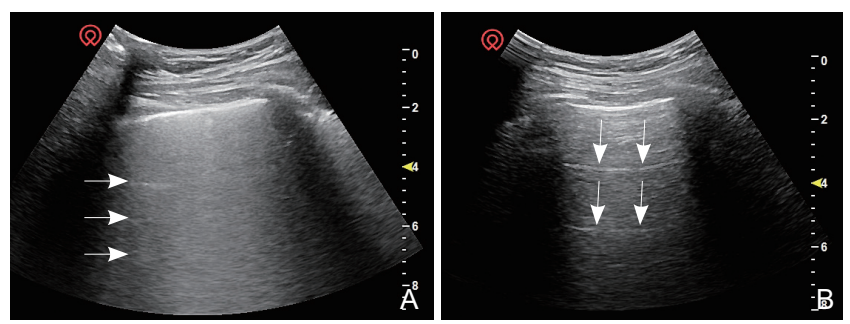

Figure 2 Remote ultrasound images of lung. (A) Case 1, a 53-year-old female, on the day of admission, ultrasound showed multiple B3-lines in both lungs, which fused into each other to form "white lung" (arrow); (B) 24 days after admission, A-line (arrow) appeared in both lungs.

\section{CT results}

On the day of admission, the chest CT of the two patients showed diffuse ground-glass shadows distributed in both lungs. They gradually dissipated after intermittent reexamination of the $\mathrm{CT}$, and finally the lesions completely dissipated. There was a high degree of consistency between ultrasound and CT findings in those two patients (Fig. 3).

\section{Laboratory test}

Laboratory tests were performed and the results were shown in Table 1 and Table 2. 

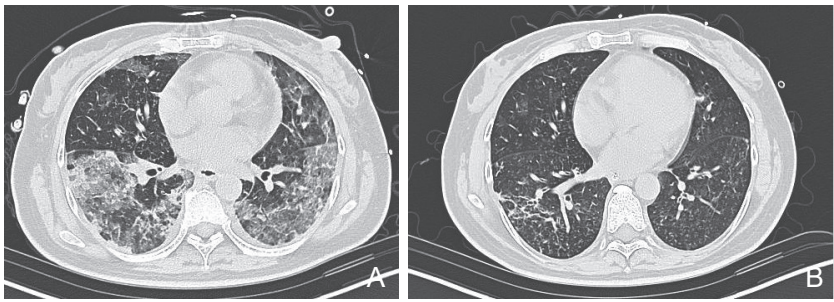

Figure 3 Plain CT scan of the lung. (A) Case 1, a -53-years old female patient; CT showed diffuse distribution of ground glass in both lungs on the day of admission; (B) Most of the ground glass density shadows disappeared 24 days after admission.

\section{Discussion}

The clinical condition of critically ill COVID-19 patients can deteriorate rapidly. It was reported that $31 \%$ of critically ill patients had heart injury, especially elderly patients with basic heart disease [5,6]. Therefore, the diagnosis and treatment of other basic diseases is also an important part of the treatment of COVID-19. The condition of severe COVID-19 patients in the isolation ward changes rapidly, which mandates fast and accurate evaluation at any time.

Table 1 Laboratory results (part 1)

\begin{tabular}{lcccccc}
\hline Day(s) after admission & 1 & 2 & 3 & 8 & 10 & 24 \\
\hline Leukocyte $\left(10^{9} / \mathrm{L}\right)$ & 11.51 & 13.46 & 16.05 & 5.9 & 5.19 & 5.25 \\
Lymphocyte $\left(10^{9} / \mathrm{L}\right)$ & 0.06 & 0.019 & 0.975 & 0.039 & 0.823 & 0.37 \\
\hline C-reactive protein $(\mathrm{mg} / \mathrm{dl})$ & 10.04 & 21.52 & 6.6 & 0.5 & 1.16 & 3.24 \\
\hline Procalcitonin $(\mathrm{ng} / \mathrm{ml})$ & 0.92 & 0.584 & 0.459 & 0.279 & 0.051 & 0.037 \\
IL-6 $(\mathrm{pg} / \mathrm{ml})$ & 491.7 & 14.45 & 48.89 & 2.85 & 12.18 & 6.65 \\
D-Dimer $(\mathrm{ng} / \mathrm{ml})$ & 3726 & 4229 & 19726 & 1412 & 1558 & 830 \\
nCOV & $(-)$ & $(-)$ & $(-)$ & $(-)$ & $(-)$ & $(-)$ \\
Antibody $\quad \operatorname{lgM}(\mathrm{mg} / \mathrm{dl})$ & 52.7 & & & 81.9 & 69.3 \\
\hline $\operatorname{lgG}(\mathrm{mg} / \mathrm{dl})$ & 1670 & & & 1270 & 1220 \\
\hline
\end{tabular}

Reference range: Leukocyte, 3.5-10; Lymphocyte, 0.20-0.40; C-reactive protein, 0-0.5; Procalcitonin, < 0.5; IL-6, 0-7; D-dimer, < 500; IgG, 700-1600; IgM, 40-230.

Table 2 Laboratory results (part 2)

\begin{tabular}{lccc}
\hline \multicolumn{1}{c}{ Day(s) after admission } & 1 & 2 & 12 \\
\hline Leukocyte $\left(10^{9} / \mathrm{L}\right)$ & 15.23 & 10.88 & 11.4 \\
\hline Lymphocyte $\left(10^{9} / \mathrm{L}\right)$ & 0.048 & 0.039 & 0.05 \\
\hline C-reactive protein $(\mathrm{mg} / \mathrm{dl})$ & 2.66 & 1.7 & 0.04 \\
Procalcitonin $(\mathrm{ng} / \mathrm{ml})$ & 0.071 & 0.159 & 0.035 \\
\hline IL-6 (pg/ml) & 20.89 & 1.5 & 1.5 \\
\hline D-Dimer $(\mathrm{ng} / \mathrm{ml})$ & 933 & 1165 & 352 \\
\hline nCOV & $(-)$ & $(-)$ & $(-)$ \\
\hline Antibody $\quad \operatorname{lgM}(\mathrm{mg} / \mathrm{dl})$ & 699 & & \\
\hline$\quad \operatorname{lgG}(\mathrm{mg} / \mathrm{dl})$ & 746 & & \\
\hline
\end{tabular}

Reference range: Leukocyte, 3.5-10; Lymphocyte, 0.20-0.40; C-reactive protein, 0-0.5; Procalcitonin, < 0.5; IL-6, 0-7; D-dimer, < 500; IgG, 7001600; IgM, 40-230.

Although chest CT is currently the main imaging modality of evaluating COVID-19 lung lesions [7], bedside ultrasound has a role in the course of COVID-19 prompt diagnosis and treatment due to its portability and non-invasiveness. In the epidemic period of COVID-19, the ICU physicians in isolation wards were the main physicians to treat patients, while the ultrasound experts entering the isolation ward would increase the chance of infection and cause excessive consumption of PPE.

Remote ultrasound plays an important role in the rational allocation of medical resources and the guarantee the quality of patient care. In our own practice, the ICU physician at the patient end has inadequate experience of performing ultrasound examination. $\mathrm{He}$ brought the portable ultrasound machine into the ICU isolation ward, and performed ultrasound examination under the supervision of ultrasound experts at remote end. We found a high degree of consistency between ultrasound and CT findings. However, the doctors on the patient side lack the training of the ultrasonic standard cross-sectional scan and need the guidance from remote experts, which prolongs the examination time. One possible solution we found was practicing before the real exam. The application of remote ultrasound in the isolation ward of COVID-19 patients can also help to save medical resources to a certain extent, make full use of remote specialist resources, and reduce the chance of 
exposure for more medical staff. With the development of $5 \mathrm{G}$ and other communication technologies, remote ultrasound has been widely used in obstetrics and gynecology, cardiac, musculoskeletal system, ocular trauma ultrasound, and focused assessment with sonography for trauma (FAST) [8-10]. In addition, for critically ill COVID-19 patients, wireless network could provide a very convenient platform for multidisciplinary consultation, collaborative diagnosis, and optimum treatment.

In summary, the application of remote ultrasound can timely monitor the changes of pulmonary findings, cardiac condition, volume state, and other organ systems. Ultrasound experts do not have to enter the isolation area to provide consultation advice guiding clinical treatment. In the special period of COVID-19, with increased number of patients and insufficient medical staffs and PPE, remote ultrasound can help alleviate the pressure and maintain the quality of care. Since there is limited research focusing on remote ultrasonography, the diagnostic performance of this remote ultrasound model needs to be further studied.

\section{Acknowledgment}

Funding was provided by Major Science and Technology Project of Hainan Province of China (No.ZDKJ2019012), Military Medical Research Program of PLA General Hospital of China (No. CX19025 and No. QNC19050), National MOST “13.5” Key Projects of China (No.2016YFC0105006), Talents Development Project of Sanya City of China (PI: Faqin Lv), and Medical and Health Science and Technology Innovation Project of Sanya City (No. 2018YW01).

\section{Conflict of Interest}

The authors have no conflict of interest to declare.

\section{References}

[1] National Health Commission of the people' s Republic of China. Diagnosis and treatment of novel coronavirus pneumonia (trial, the seventh version) [EB/OL]. (2020-03-03) [2020-03-04]. Available from: http://www.nhc.gov.cn/yzygj/s7653p/202003/46c9294a7dfe4ce f80dc7f5912eb1989.shtml.

[2] Li M, Lei P, Zeng B, Li Z, Yu P, Fan B, et al. Coronavirus disease (COVID-19): spectrum of ct findings and temporal progression of the disease. Acad Radiol 2020: S1076-6332(20)30144-6.

[3] National Health Commission Capacity Building and Continuing Education Center; The War trauma and Emergency Ultrasound Committee of Medical Ultrasound Equipment Society of CAME; Tele- and Mobile- Ultrasound Subcommittee of Medical Ultrasound Equipment Society of CAME. Chinese expert consensus on critical ultrasound application for patients with COVID-19. Chinese Journal of Critical Care Medicine 2020; 3: 185-195. [In Chinese]. Available from: http://111.40.160.75:802/CN/abstract/abstract5262.shtml.

[4] Liu Y, Huang Y, Lv F, Li T. Progress of tele-ultrasound technology. Chinese Journal Medical Ultrasound (Electronic Edition) 2019; 16: 244246. [In Chinese] DOI: 10.3877/cma. j.issn.1672-6448.2019.04.002.

[5] Wang Z, Yang B, Li Q, Wen L, Zhang R. Clinical Features of 69 Cases with Coronavirus Disease 2019 in Wuhan, China. Clin Infect Dis. 2020 Mar 16: ciaa272. DOI: 10.1093/cid/ciaa272.

[6] Lian J, Jin X, Hao S, Cai H, Zhang S, Zheng L, et al. Analysis of Epidemiological and Clinical features in older patients with Corona Virus Disease 2019 (COVID-19) out of Wuhan. Clin Infect Dis. 2020 Mar 25: ciaa242. DOI: 10.1093/cid/ciaa242.

[7] Li K, Fang Y, Li W, Pan C, Qin P, Zhong Y, et al. CT image visual quantitative evaluation and clinical classification of coronavirus disease (COVID-19). Eur Radiol. 2020 Mar 25. DOI: 10.1007/ s00330-020-06817-6

[8] Hishitani T, Fujimoto Y, Saito Y, Sugamoto K, Hoshino K, Ogawa $\mathrm{K}$. Accuracy of telediagnosis of fetal heart disease using ultrasound images transmitted via the Internet. Pediatr Int 2014, 56: 289-291.

[9] Dyer D, Cusden J, Turner C, Boyd J, Hall R, Lautner D, et al. The clinical and technical evaluation of a remote telementored telesonography system during the acute resuscitation and transfer of the injured patient. J Trauma 2008, 65: 1209-1216.

[10] Liu Y, Lv F, Li T. The advent of 5G ultrasound: Application and progress of remote ultrasound. Chinese Journal Medical Ultrasound (Electronic Edition) 2019; 16: 241-243. [In Chinese] DOI: 10.3877/ cma.j.issn.1672-6448.2019.04.001. 